Cahiers $d u$ MONDE RUSSE

\section{Cahiers du monde russe}

Russie - Empire russe - Union soviétique et États indépendants

$57 / 4 \mid 2016$

Varia

\title{
Yanni Kotsonis, States of Obligation - Taxes and Citizenship in the Russian Empire and Early Soviet Republic
}

\author{
Juliette Cadiot
}

\section{(2) OpenEdition}

1 Journals

\section{Édition électronique}

URL : http://journals.openedition.org/monderusse/10021

DOI : 10.4000/monderusse. 10021

ISSN : $1777-5388$

Éditeur

Éditions de l'EHESS

\section{Édition imprimée}

Date de publication : 1 octobre 2016

Pagination : 931-934

ISBN : 978-2-7132-2542-0

ISSN : $1252-6576$

\section{Référence électronique}

Juliette Cadiot, "Yanni Kotsonis, States of Obligation - Taxes and Citizenship in the Russian Empire and Early Soviet Republic », Cahiers du monde russe [En ligne], 57/4 | 2016, mis en ligne le 01 octobre 2018, Consulté le 25 septembre 2020. URL : http://journals.openedition.org/monderusse/10021 ; DOI : https://doi.org/10.4000/monderusse.10021 
why this was such a controversy (the political climate was extremely polarised at this point and only nine months later the prime minister, Stolypin, was assassinated), Fishzon incorporates the story into her narrative of fan letters to the celebrity singer.

More could also be made of gender politics. While gender relations underpin the entire work, Fishzon does not pay attention to the actual changes in Russian society in the last fifty years of the Imperial regime, in which women attained access to education at all levels, women's employment outside the home increased and, from 1905, campaigns for women's suffrage (as part of the liberal and revolutionary movements for political change) developed. The more visible women became, whether as teachers, doctors or celebrity actresses, the more women were subjected to misogyny and satirical lampoons. More acknowledgement of this would have added to the discussion of women as consumers of culture and perhaps shed more light on those adoring fans, whose letters have been preserved in the archives and which she uses to great effect.

There is a wealth of material in this book, much of it very entertaining and informative. There is some repetition between chapters and some incoherent organisation, as though the chapters had originated as separate articles. Finally, a plea to the publishers: this book would have been so much easier to read if the extensive endnotes, some of them providing important information and comment, had been incorporated into the text as footnotes.

\section{Linda Edmondson \\ University of Birmingham, UK}

\begin{tabular}{cccccccccccccccccccc}
\hline & $P$ & $E$ & $R$ & $I$ & $O$ & $D$ & $E$ & & $S$ & $O$ & $V$ & $I$ & $E$ & $T$ & $I$ & $Q$ & $U$ & $E$ \\
& $E$ & $T$ & & $P$ & $O$ & $S$ & $T$ & $S$ & $O$ & $V$ & $I$ & $E$ & $T$ & $I$ & $Q$ & $U$ & $E$
\end{tabular}

\section{Yanni KOTSONIS}

\section{States of Obligation}

\section{Taxes and Citizenship in the Russian Empire and Early Soviet Republic}

Toronto - Buffalo - London : University of Toronto Press, 2014, 504 p.

À travers l'étude de l'imposition (du XVIII ${ }^{\mathrm{e}}$ siècle jusqu'à la collectivisation), cet ouvrage somme constitue une tentative particulièrement réussie et sophistiquée d'histoire politique de l'État moderne en Russie. Le livre comble un manque en inscrivant cet empire dans une histoire comparative des systèmes de taxation. L'État, l'économie et l'individu constituent les trois concepts convoqués par Kotsonis, leur signification et relations mutuelles évoluant profondément pendant la période allant de la fin du servage au Grand Tournant stalinien qui constitue le cœur de l'ouvrage.

L'auteur restitue les nombreux débats et les tentatives de révision du système fiscal menées au sein du gouvernement, du ministère des Finances, dans les 
commissions aux impôts,... Les contemporains attribuaient à l'impôt un pouvoir majeur de transformation économique, sociale et politique. Ils voyaient dans l'évolution de la fiscalité le moyen de répondre à des questions tant techniques de connaissance et mise en valeur du pays qu'éthiques et politiques de justice et de transformation des hiérarchies sociales. Enfin, un meilleur impôt permettait d'agir sur le mieux-être économique du pays en renforçant la capacité financière de l'État. L'enjeu de la construction d'une machine étatique et de l'extension d'une bureaucratie nécessaire à la collecte de l'impôt supposait une amélioration progressive de la capacité de prévision et de calcul de l'État, permettant en retour la bonne élaboration d'un budget. Cette dernière exigence est liée à l'émergence du concept moderne d'économie politique. En offrant un miroir de l'activité du pays, l'impôt permettait d'évaluer la richesse nationale et, idéalement, de construire une loi budgétaire en lien avec l'économie réelle dont elle était le reflet. Néanmoins, dans ce processus, la Russie impériale, libérale puis bolchevique, se heurta à la difficulté de connaître le pays.

La question de la manière d'imposer les paysans se posa sur l'ensemble de la période étudiée. À la fin du servage, alors que la population était aux 4/5 paysanne, les informations sur la richesse des campagnes étaient minimales. À partir des années 1890, la question de la charge de l'impôt pesant sur des paysans récemment libérés (qui devaient s'acquitter d'une double taxe : impôt foncier et indemnité de rachat des terres) anima le débat public. Pourtant, comme le montre Kotsonis, en 1913, l'impôt paysan ne représentait que $1 \%$ du revenu total de l'État. La question tant débattue du fardeau fiscal pesant sur les paysans constituait un faux problème, mais la taxation paysanne fut un échec constant. Le monde des campagnes resta inconnu, un territoire isolé dont l'imposition se fit de manière arbitraire. L'impôt y faisait l'objet d'une répartition inégalitaire, injuste, domaine de la responsabilité collective et des châtiments corporels, avant que, avec les guerres et les révolutions, il ne soit remplacé par les réquisitions obligatoires, forcées et particulièrement brutales.

Yanni Kotsonis s'intéresse à la relation directe que l'État moderne tenta de nouer avec ses sujets à travers le passage de l'impôt de répartition (rasklad, razvertska) - système dans lequel une charge publique peu précisée est partagée par des collectivités (et fermiers généraux) -, à l'impôt de quotité (obloženie). Ce dernier exige une estimation précise de l'activité économique de chacun, permettant d'équilibrer les demandes en termes d'impôts et de connaître la richesse du pays. Au terme du processus, de par leur (honnête) déclaration de propriété, profit et revenu, les individus éprouvent le sens de leur obligation envers l'État. Dans ce schéma, l'impôt, plus encore que le vote, les transforme en citoyens. Dans ce récit, l'impôt de quotité devient la métaphore (ou la métonymie) de la construction d'une relation directe mettant face à face dans une relation disproportionnée l'individu et l'État.

Yanni Kotsonis décrit l'échec du projet d'instauration de l'impôt de quotité en Russie. L'absence de cadastre, le manque général de statistiques et d'informations, sans parler des troubles, ajournèrent sans cesse son application dans les campagnes. L'impôt de quotité supposait une connaissance suffisamment précise de ce que 
chacun gagnait, possédait et pouvait donner pour contribuer à l'effort commun. Finalement la quotité se limita aux villes. À partir des années 1890, les citadins s'acquittèrent d'une taxe sur le commerce (1885), puis sur les appartements (1893). L'introduction de l'impôt sur le revenu fut discutée en Russie dès 1907 et adoptée en 1916. Mais sur l'ensemble de la période, ce furent le monopole de l'alcool et les impôts indirects qui fournirent l'essentiel des revenus de l'État marquant l'échec de la tentative d'intégration de la population paysanne russe.

Si l'avancée de l'idée de quotité constitue un des fils majeurs de son analyse, Yanni Kotsonis ne s'arrête pas à ce domaine. Il écrit l'histoire de l'ensemble de l'évolution de l'impôt dans la durée, ainsi que les pratiques locales de collecte. Les impôts sur la propriété, sur le commerce, les impôts indirects, ou même les taxes en jours de travail forcé, font l'objet d'une analyse fouillée.

Présentant les continuités qui lient les projets mis en place par les ministères des Finances et l'administration des impôts sous l'Empire, le Gouvernement provisoire et les bolcheviks, Kotsonis souligne la pérennité des personnels. $90 \%$ des membres du commissariat aux Finances et 2/3 des inspecteurs des impôts des années 1920 travaillaient dans la bureaucratie impériale. Si la majeure partie de la propriété avait été nationalisée après Octobre (en été 1918, le ministère des Finances s'inquiétait que la nationalisation ne fût en train de détruire l'objet de l'estimation), dans les années 1920, le gouvernement soviétique fit un effort délibéré pour retourner à ce qui avait existé avant 1914, prenant pour modèle les revenus de l'État de 1913. Dans les villes, divers projets d'impôt sur le revenu furent appliqués, alors que dans les campagnes, des levées (souvent en nature) par répartition continuaient. Le budget du nouvel État se fondait, comme avant la guerre, d'abord sur les taxes indirectes, ou encore sur un système de licence pour taxer les opérations commerciales, les droits de succession et le monopole de vente de la vodka. La quête d'information reprise par les bolcheviks se heurta aux mêmes difficultés techniques que celles rencontrées par leurs prédécesseurs.

Cet ouvrage permet de suivre de manière claire et stimulante une histoire technique, complexe, de différentes formes d'imposition et de taxation au cours d'une période cruciale de l'avènement d'un État fort, moderne et autoritaire, pourtant incapable de fonder un système de quotité, d'estimer précisément la richesse de sa population et d'introduire un impôt juste. Soulignant la division entre ville et campagne et la poursuite, commune aux modernisateurs de l'Empire comme aux bolcheviks, d'une relation non médiatisée entre les citoyens et l'État, l'ouvrage ouvre une série de pistes de réflexion sur la nature du pouvoir politique en Russie.

Tout en décrivant l'échec des tentatives des fonctionnaires d'État de connaître la richesse des habitants, voire de comprendre l'organisation de l'économie réelle, l'auteur n'articule pas toujours suffisamment dans son récit la radicalité des pensées réformatrices et l'existence de mécanismes sociaux qui échappent largement à l'État. Kotsonis conclut que les dirigeants ne purent finalement accéder à la richesse des paysans qu'à travers la violence de la spoliation. Il décrit peu les moments où l'administration du fisc fut capable de négocier pour garantir des revenus à l'État, par exemple dans le cadre de la responsabilité collective. Cet aspect constitue un 
domaine de recherche sur les formes de relations liant l'État et la population qui pourrait être développé dans le cadre d'un projet s'appuyant sur le travail déjà réalisé. À cela s'ajoute l'analyse des moments où les individus ne reconnurent pas leurs obligations envers l'État et tentèrent de les négocier. En inversant le point de vue, en étudiant les phénomènes d'évasion fiscale, de refus des impôts, voire de détournements économiques pour éviter la taxation, une telle analyse offrirait le pendant à un récit historique fondé sur les projets de technocrates modernisateurs.

\section{Juliette Cadiot}

CERCEC, EHESS

\section{Malen kij čelovek i bol`šaja vojna v istorii Rossii, seredina XIX-seredina XX v. Materialy meždunarodnogo kollokviuma (Sankt Peterburg, 17-20 ijunja 2013 g.)}

[Les petites gens et la grande guerre dans l'histoire de la Russie, milieu du XIX ${ }^{\mathrm{e}}$ milieu du Xx : Actes du colloque international, (Saint-Pétersbourg, 17-20 juin 2013)] Saint-Pétersbourg : Nestor, 2014, 572 p.

В последней трети 20 в. под влиянием общей тенденции антропологизации исторического знания, осмысления «лингвистического», «интерпретативного» и других «поворотов» и интеллектуальных вызовов постструктурализма фокус исследовательского интереса в истории заметно сместился к изучению социокультурных и субъективных аспектов социально-исторических процессов ${ }^{1}$. На Западе эта тенденция стала проявляться уже в 1970-е гг., в России - с 1990-х гг, когда российская историческая наука освободилась от монополии марксистской методологии.

Применительно к военной истории новыми, активно осваиваемыми тематическими направлениями на Западе, а затем и в России, стали поведенческие практики, психология комбатантов и мирного населения; повседневность фронта и тыла; формирование и эволюция памяти о войне; проявления религиозной и этнической идентичности в условиях войн; дискурс участников и современников войн и др. ${ }^{2}$ В российской историографии помимо общего сдвига в проблематике истории войн произошло оформление особого исследовательского направления - «военно-исторической антропологии», лидером которого стала признанный специалист в этой области Е.С. Сенявская ${ }^{3}$.

Очевидная смена исследовательских приоритетов проявилась в переходе от событийной истории к изучению опыта войны ${ }^{4}$. Как отмечает лидер данного направления Д. Байрау, «понятие “опыт” включает в себя субъективные категории: разговоры, современные и более поздние рассказы и воспоминания - в общем, дискурс участников и их потомков о войне. Все эти компоненты образуют индивидуальный и коллективный опыт» ${ }^{5}$. Российские сторонники 